\title{
A modified fractal texture image analysis based on grayscale morphology for multi-model views in MR Brain
}

\author{
Usha R, Perumal K
}

Department of Computer Applications, School of Information Technology, Madurai Kamaraj University, Madurai, India

\begin{tabular}{|c|c|}
\hline Article Info & ABSTRACT \\
\hline Article history: & This paper presents a modified fractal texture feature analysis with the use of \\
\hline Received Mar 15, 2020 & $\begin{array}{l}\text { grayscale image morphology for automatic image classification of different } \\
\text { views in MR brain images into normal and abnormal. This main contribution }\end{array}$ \\
\hline Revised May 15, 2020 & of this approach is a reduction of the total number of a threshold value, and \\
\hline Accepted Jun 23, 2020 & $\begin{array}{l}\text { the number of image decomposition, in which only the number of extract } \\
\text { threshold value two or three are enough for tumor region extraction }\end{array}$ \\
\hline Keywords: & $\begin{array}{l}\text { compared to four or more is required in the previous method of SFTA } \\
\text { (segmentation based fractal texture analysis). This is achieved by pre- }\end{array}$ \\
\hline Classification & processing of hierarchical transformation technique (HTT), which make use \\
\hline Ensemble bagged tree & $\begin{array}{l}\text { of morphological image transformations with the desired structural element. } \\
\text { From this decomposed images, mean, area, fractal dimension and selective }\end{array}$ \\
\hline Fractal texture & shape features are extracted and fed into KNN and ensemble bagged tree \\
\hline Image segmentation & classifiers. Finally, some of the post-processing is handled for tumor region \\
\hline Kth nearest neighbour & extraction and tumor cells computation. It is found that this proposed \\
\hline Morphological operations & approach has superior results in the segmentation of diseased tissue from \\
\hline Tumor cell computation & $\begin{array}{l}\text { normal tissue and the prediction of image classes in terms of accuracy with } \\
\text { the less number of threshold extraction and image decomposition rather than } \\
\text { the SFTA algorithm. }\end{array}$ \\
\hline
\end{tabular}

This is an open access article under the CC BY-SA license.

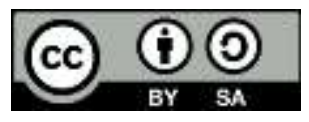

Corresponding Author:

R. Usha

Department of Computer Applications

School of Information Technology, Madurai, India

Email: usha.resch@gmail.com

\section{INTRODUCTION}

In image processing and computer vision, a feature is a piece of interesting information that indicates some quantifiable attribute of an image to achieve the computational task. The extracted features from an image are much lesser dimension than the original image that dimensionality reduction reduces the issues of the bulk of image processing. Generally, image features are categorized into two types like local and global features. Local features are calculated over the outcomes of image band separation when image segmentation or the process of edge detection [1]. And the global features are computed on the entire portion of an image or regular sub-regions in an image. In this way, the global feature texture is an important one to analyse the regional descriptor that useful in the process of pattern matching or retrieval process [2]. In this work, or the recognition and image patterns, the segmentation process has carried out to extract some significant intended features from an image. In that aspect, many researchers are introduced several techniques to analyse MR brain images. Amulya E.R et al [3] introduced the texture feature-based classification of different views of MR brain images with the use of GLCM and SVM (support vector machine) techniques. Pratima Purushottam Gumaste and Vinayak K. Bairagi [4] developed the hybrid 
technique of advanced textural feature extraction to detect the tumor tissues from healthy tissue of different views of MR brain slices with use of support vector machine. In addition to, Marwan A. A. Hamid and Najeed Ahmed Khan [5] discussed that the method of DWT and PCA (principal component analysis) for dimensionality reduction followed by GLCM feature based SVM classification to analyse the MR brain tumors. Consequently, Alceu Ferraz Costa et al., [6] proposed the algorithm of segmentation based fractal texture analysis (SFTA) that is most frequently used in many image-based applications for the purpose of image segmentation and texture feature extraction.

For instance, M. Anju Krishna et al., [7] presented the Liver tumor segmentation and feature extraction using the SFTA method. Similarly, Spandana Paramkusham et al [8] have carried out on different feature extraction approaches of rotation invariant local frequency (RILF), local binary pattern (LBP), and SFTA (segmentation based fractal texture analysis) for breast abnormality detection using SVM classification. Saban Ozturk and Bayram Akdemir [9] were presented the comparison of various feature extraction algorithms of GLCM, LBP, LBGLCM, GLRLM and SFTA for automatic histopathological image analysis. From these comparisons, the SFTA feature extraction approach achieved the maximum success rate of accuracy. However, Jude Hemanth et al [10] have mentioned that the image pre-processing step has an important role for further image analysis steps of image segmentation, feature extraction [11], and classification. According to the literature study, many researchers are presented related to pre-processing based image analysis. Richard Alan Peters [12] suggested that the mathematical morphological image transformations are very helpful to analyse the images in different image processing techniques like noise suppression, edge detection, segmentation, feature extraction, shape recognition, texture image analysis, and image restoration etc. $\mathrm{R}$ Usha et al [13] discussed the combination of morphological transformation and fractal texture-based image segmentation technique to analyse the MR brain tumor images. G. Bala Maalinii and Anand Jatti were presented [14] the combined approach of morphological reconstruction and thresholding approaches for MR brain tumor detection, which also reduced the algorithm size in an effective manner.

Furthermore, S. Naganandhini et al [15] have introduced the combined approach of threshold and morphological operations based brain image segmentation technique for skull stripping. Andrei C. Jalba et al., [16] were introduced the morphological hat-transform scale-spaces based pattern classification approach for diatom images and Brodatz texture image set. A watershed algorithm based texture image analysis leads to the problem of over-segmentation. For that, D. Suvarna et al., [17] included the morphological operations of opening and closing in a watershed algorithm for various textures image analysis. Ankur Jyoti Das et al., [18] were carried out that the combination of morphological operations and K-means based segmentation approach for automatic detection of MR brain tumor. As with R. Radhakrishnan et al [19] suggested that the extraction of fractal dimension feature through the morphological decomposition operations of dilation and erosion provide better outcomes. Yanpeng Wu et al., [20] mentioned that the traditional watershed algorithm is very poorer in a segment the object in distinct size with the use of single threshold value. According to the reason, the additional morphological dilation and erosion operations were incorporate with a watershed algorithm for better image segmentation. Yingbo Liang and Jian Fu [21] have discussed image morphology and total variation model based watershed segmentation and concluded that the traditional problem of oversegmentation was avoided in a watershed algorithm. Yu Wang et al., [22] introduced morphological top-hat transformation based segmentation and feature extraction approach for SVM classification on mice liver fibrosis microscopic images in less computational time. Dhananjay Bhaskar et al., [23] introduced the region growing and morphological transformation based feature was extracted to classify the cell microscopic images. Nelson Luis Duranona Sosa et al., [24] mentioned that the morphological transformations like erosion and dilation operations act as texture descriptor in RGB images. Similarly, morphological image transformation was applied by many researchers to analyse the images.

In this paper, the mathematical morphology has used for both purpose of image segmentation, and feature extraction to analyse the different views of MR brain slices. The fractal texture analysis algorithm of the SFTA [6] technique is considered to be one of feature extraction approaches for further classification. The conventional SFTA algorithm works better than the GLCM, Haralick features. However, it takes the numerous number of a threshold value, image decomposition to extract the affected parts of the brain and feature classification. In this method, the morphological transformation based fractal texture analysis approach has discussed with the use of Kth nearest neighbour and ensemble bagged tree classifier algorithms to analyse the different views of MR brain images.

\section{MR BRAIN IMAGE SET}

The multi-model view of MR brain image samples are gathered from Madurai Rajaji Government hospital and KG Advanced scan center, Madurai, Tamilnadu together of 525 brain slices normal and 
abnormal classes. From this 240 (168 images are abnormal and 72 images are normal) images are for axial view, 187 images in coronal view (123 for abnormal and 64 for normal), and 98 images are available in sagittal view (50 abnormal and 48 normal) of MR brain. From this $60 \%$ images are allocated for training and remaining images are taken for testing in each view of normal and abnormal classes. The sample images of two different classes in various views of MR brain has illustrated in Figure 1.

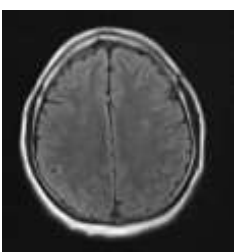

(a)

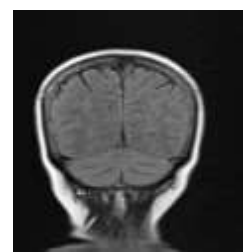

(b)

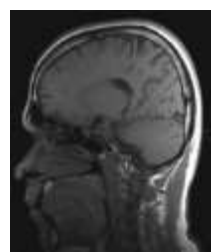

(c)

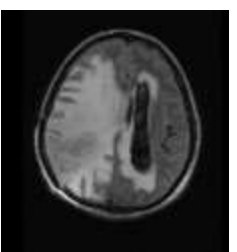

(d)

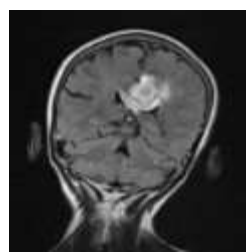

(e)

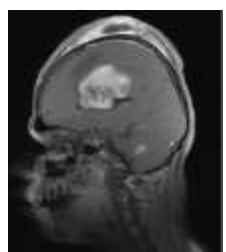

(f)

Figure 1. Normal and abnormal images of different views of MR brain. axial (a) normal and (d) abnormal; coronal (b) normal and (e) abnormal; sagittal (c) normal and (f) abnormal

\section{RESEARCH METHOD}

In this paper, morphological transformation based fractal texture analysis method is introduced to detect the brain abnormality and to extract the significant texture features in different views of MR brain images. This proposed method of MR brain image classification has outlined in Figure 2. In this approach, the different views of MR brain images are taken as an input this system. Primarily, the morphological transformation operations are applied in pre-processing phase. Then, the fractal texture analysis process is used for image segmentation and texture feature extraction. The extracted texture features are fed into feature classification algorithms such as KNN and ensemble bagged tree, which are classifies the unknown test image data with use of known set of trained predefined categories of class labels. Finally, the tumor regions are extracted from the recognized class of abnormal images to calculate the defected cells of brain image.

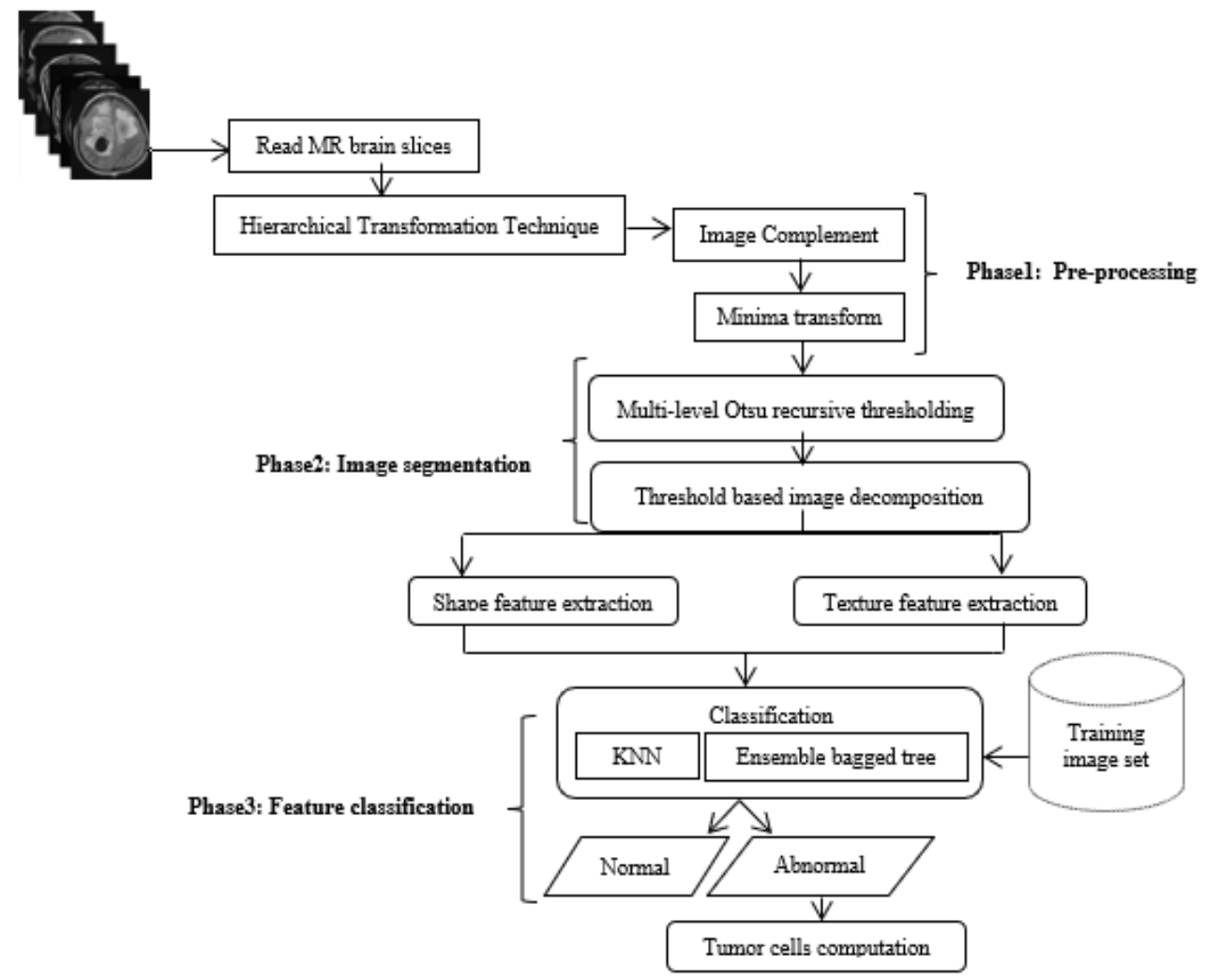

Figure 2. Morphological transformation based fractal texture analysis in different views of MR brain image 


\subsection{Pre-processing}

During this first stage, the hierarchical transformation technique [25] is applied to enhance the image contrast and improve the image quality with use of top-hat and bottom-hat image transforms. This method incorporates the essential steps of optimum disk-shaped structural element selection which helps to select the suitable size of SE value for a specific brain image. Because, the size of disk-shape mask is different from one image to another. Consequently, the image complement operation is utilized on hierarchical transformed image that are helps to highlight the defected cells of brain images. This operation is mathematically defined by the equation of,

$$
U(i, j)=\neg(V(i, j))
$$

Where, $\mathrm{V}(\mathrm{i}, \mathrm{j})$ and $\mathrm{U}(\mathrm{i}, \mathrm{j})$ are denote the hierarchical transformed and complementary image respectively. Finally, the minima transform operation has used to avoid the issue of over-segmentation [26]. The mathematical definition of H-minima transform is calculated by the equation of,

$$
M_{m}(c)=T_{c}(c+m)
$$

Here, $\mathrm{c}, \mathrm{m}$, and $\mathrm{T}$ indicate the inverse distance map of clustered nuclei, depth, and reconstruction and erosion operators respectively.

\subsection{Segmentation and feature extraction}

The fractal analysis of texture algorithm [6] has divided into two different stages like (i) threshold based image decomposition and (ii) texture feature extraction. Initially, the multi-level Otsu thresholding technique has applied to each image regions recursively, until the total number of threshold is found. The total number of threshold is decided by the end user. In this work, threshold size is initialized as 2 . Threshold based image decomposition has mathematically expressed by the equation of,

$$
I_{d}(\mathrm{p}, \mathrm{q})=\left\{\begin{array}{cc}
1, & \text { if } t_{\text {lower }}<I_{g}(p, q) \leq t_{\text {upper }} \\
0, & \text { otherwise }
\end{array}\right.
$$

Where, $t_{\text {lower }}$ and $t_{\text {upper }}$ indicate the lower and upper threshold values. From these decomposed process of each segmented images, the mean, area, boundaries fractal dimension feature and addition to, shape features of solidity, perimeter, and centroid features are extracted. The extracted feature vector of unknown test image data is used for further classification with use of known set of training set.

\subsection{Feature classification}

Classification is one of the methods to recognize the relevant class label of unknown test sample with known set of trained class labels. In this paper, KNN and ensemble bagged tree machine learning algorithms are used to classify the image labels. In KNN algorithm, the features of testing set are classified by distance measure of Euclidian distance from its neighbour in the brain image's features that are assigned to the class. Here, the performance KNN algorithm has estimated by the choice of ' $\mathrm{K}$ ' and distance metric [27]. The mathematical definition of Euclidian distance is expressed as,

$$
\mathrm{D}(\mathrm{a}, \mathrm{b})=\sqrt{\left(\left(a_{1}-b\right)^{2}+\left(a_{2}-b_{2}\right)^{2}+\cdots+\left(a_{n}-b_{n}\right)^{2}\right)}
$$

where, $\mathrm{a}$ and $\mathrm{b}$ are represent the feature points of test and trained data.

The formation of the bag with the decision tree learners are refereed as Bagged Tree that is one of the best ensemble machine learning algorithms. The feature sampling decomposition is formulated by the technique of bootstrap aggregation, which is: select the $\mathrm{M}$ out of $\mathrm{M}$ sub training set with feature replacement for each new learner. The formation of decision tree for each sub training set is depends on the attributes of gini index, entropy and information gain. The definition of gini index can be defined as,

$$
\begin{aligned}
& g=1-\sum_{j=1}^{m} X_{j}{ }^{2} \\
& \text { Here, } \quad X_{j}=\frac{\text { Total number of records in } j^{\text {th }} \text { class }}{\text { Total number of records }(m)}
\end{aligned}
$$

And every tree in the ensemble can randomly select predictors for decision splits which method helps to increase the accuracy of bagged trees. 


\subsection{Tumor cells computation}

The recognized abnormal brain images are moved into next sub-sequent phase of area opening which helps to remove the small objects from binary image. From this extracted tumor portion, the number of affected cells is also calculated using binarization technique. The segmented binary images have two values such as white (1) and black (0) pixels. Initially, the total number of zero (0) and non-zero pixels (1) in an image $(\mathrm{X})$ can be computed by the equation of,

$$
X=\sum_{p=0}^{255} \sum_{q=0}^{255}[s(0)+s(1)]
$$

Image pixels=width $(\mathrm{p}) \times$ height $(\mathrm{q})=256 \times 256$. The variable $\mathrm{I}, \mathrm{s}(0)$ and $\mathrm{s}(1)$ are image, black and white pixels respectively. The total number of white pixel (wp) in image $(\mathrm{X})$ has expressed by:

$$
w p=\sum_{p=0}^{255} \sum_{q=0}^{255}[s(1)]
$$

Here, $w p=$ number of white pixels and the value of 1 pixel $=0.264 \mathrm{~mm}$. Then, area of tumor computation has illustrated as:

$$
T_{\text {area }}=[(\sqrt{w p}) * 0.264] \mathrm{mm}^{2}
$$

\section{RESULTS AND DISCUSSION}

The collective MR brain images in training and testing set are pre-processed using morphological image transformation, which are shown in Figure 3 with use of selective image sample.

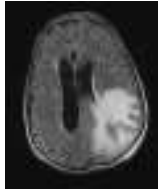

(a)

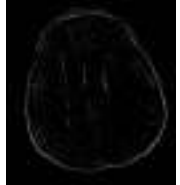

(b)

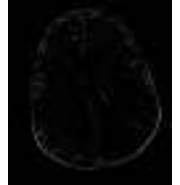

(c)

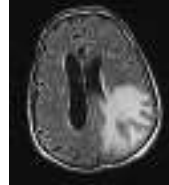

(d)

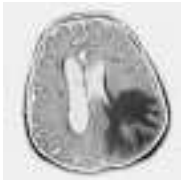

(e)

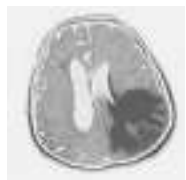

(f)

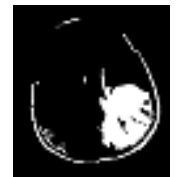

(g)

Figure 3. The morphological image transformation based fractal texture image segmented results for axial abnormal (a) Original image (b) top-hat (c) bottom-hat transformed (d) enhanced image (e) complementary image (f) minima transformed image (g) segmented image

The fractal texture image segmentation results of morphological transformed image Figure 3 (f) are presented in Figure 4.

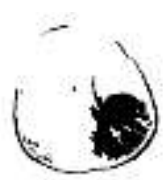

(a) binary 1 Threshold $(\mathbf{T}(\mathbf{1})=\mathbf{0 . 5 3 5 2}$

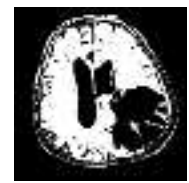

(a) binary 3

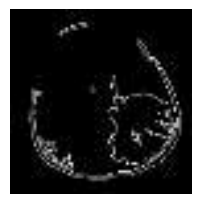

(b) border 1

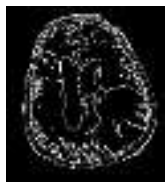

(b) border 3

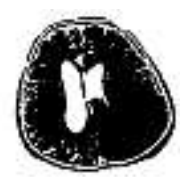

(a) binary 2

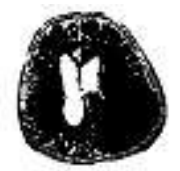

(a) binary 4

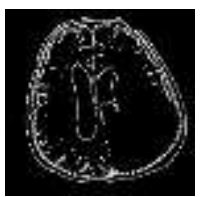

(b) border 2

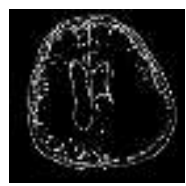

(b) border 4

Threshold $\left(t_{\mathrm{l}}=0.5352\right.$ and $\left.t_{\mathrm{u}}=0.7617\right) \quad$ Threshold $\left(t_{1}=0.7617\right.$ and $\left.t_{u}=1.0000\right)$

Figure 4. Morphological transformation based texture image segmented results for axial abnormal MR brain 
In fractal texture analysis, if the number of extracted threshold is 2 , the total number of segmented image will be as $n \times 2$. Then, boundary region are also extracted from each binary segmented images. As mentioned in Figure 4, the decomposed results of $\mathrm{T}(1)=0.5352$ has considered as segmented results. From this image, the tumor region are extracted, which is illustrated in Figure 5 as follows,

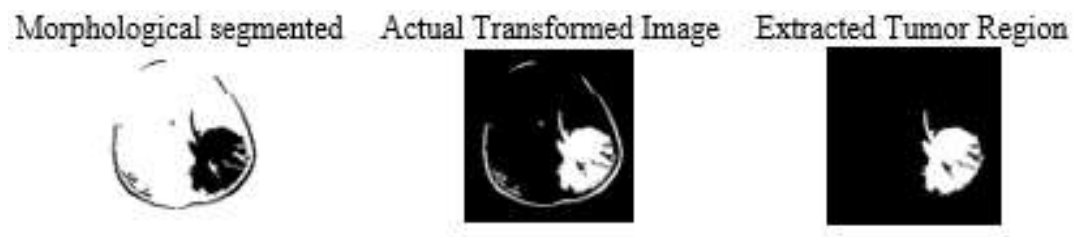

Figure 5. Tumor region extraction from the segmented abnormal binary image

From these segmented binary images, the features of mean, area, boundary fractal dimension and other shape features of solidity, perimeter, and centroid are extracted. This process is applied to both training and testing set of MR brain image data in image dataset. The classification results of this proposed feature extraction technique based ensemble bagged tree and KNN classification results are presented in Table 1 and Table 2 respectively.

Table 1. Confusion matrix for morphological transformation based fractal texture analysis of MR brain with use of KNN classifier

\begin{tabular}{|c|c|c|c|c|c|c|c|c|c|c|c|}
\hline \multicolumn{4}{|c|}{ Axial } & \multicolumn{4}{|c|}{ Coronal } & \multicolumn{4}{|c|}{ Sagittal } \\
\hline \multirow{4}{*}{ Target } & & \multicolumn{2}{|c|}{ Output } & & & \multicolumn{2}{|c|}{ Output } & \multirow{4}{*}{ Target } & & \multicolumn{2}{|c|}{ Output } \\
\hline & & $\mathrm{O}(0)$ & $\mathrm{O}(1)$ & & & $\mathrm{O}(0)$ & $\mathrm{O}(1)$ & & & $\mathrm{O}(0)$ & $\mathrm{O}(1)$ \\
\hline & $\mathrm{T}(0)$ & 62 & 6 & Target & $\mathrm{T}(0)$ & 41 & 8 & & $\mathrm{~T}(0)$ & 14 & 6 \\
\hline & $\mathrm{T}(1)$ & 4 & 25 & & $\mathrm{~T}(1)$ & 6 & 20 & & $\mathrm{~T}(1)$ & 2 & 17 \\
\hline
\end{tabular}

Table 2. Confusion matrix for morphological transformation based fractal texture analysis of MR brain with use of ensemble bagged tree classifier

\begin{tabular}{|c|c|c|c|c|c|c|c|c|c|c|c|}
\hline \multicolumn{4}{|c|}{ Axial } & \multicolumn{4}{|c|}{ Coronal } & \multicolumn{4}{|c|}{ Sagittal } \\
\hline \multirow{4}{*}{ Target } & & Output & & \multirow{4}{*}{ Target } & & Outpu & & \multirow{4}{*}{ Target } & \multicolumn{3}{|c|}{ Output } \\
\hline & & $\mathrm{O}(0)$ & $\mathrm{O}(1)$ & & & $\mathrm{O}(0)$ & $\mathrm{O}(1)$ & & & $\mathrm{O}(0)$ & $\mathrm{O}(1)$ \\
\hline & $\mathrm{T}(0)$ & 56 & 12 & & $\mathrm{~T}(0)$ & 37 & 12 & & $\mathrm{~T}(0)$ & 12 & \\
\hline & $\mathrm{T}(1)$ & 8 & 21 & & $\mathrm{~T}(1)$ & 7 & 19 & & $\mathrm{~T}(1)$ & 3 & 16 \\
\hline
\end{tabular}

From this formation of confusion matrix, the classification results of sensitivity, specificity, accuracy, and MCC measures are also computed. The mathematical definition of these classification measures is given in following equation as:

$$
\begin{aligned}
& \text { Sensitivity }=\frac{T P}{(T P+F N)} \times 100 \\
& \text { Specificity }=\frac{T N}{(T N+F P)} \times 100 \\
& \text { Accuracy }=\frac{(T P+T N)}{(T P+F P+T N+F N)} \times 100 \\
& M C C=\frac{((T P \times T N)-(F P \times F N))}{\sqrt{((T P+F P)(T P+F N)(T N+F P)(T N+F N))}}
\end{aligned}
$$

The prediction of positive and negative image samples, accuracy, and the classification quality measure of MCC are also graphically presented in Figure 6. Tumor cells computation of sample axial view in MR brain image as shown in Figure 7. 


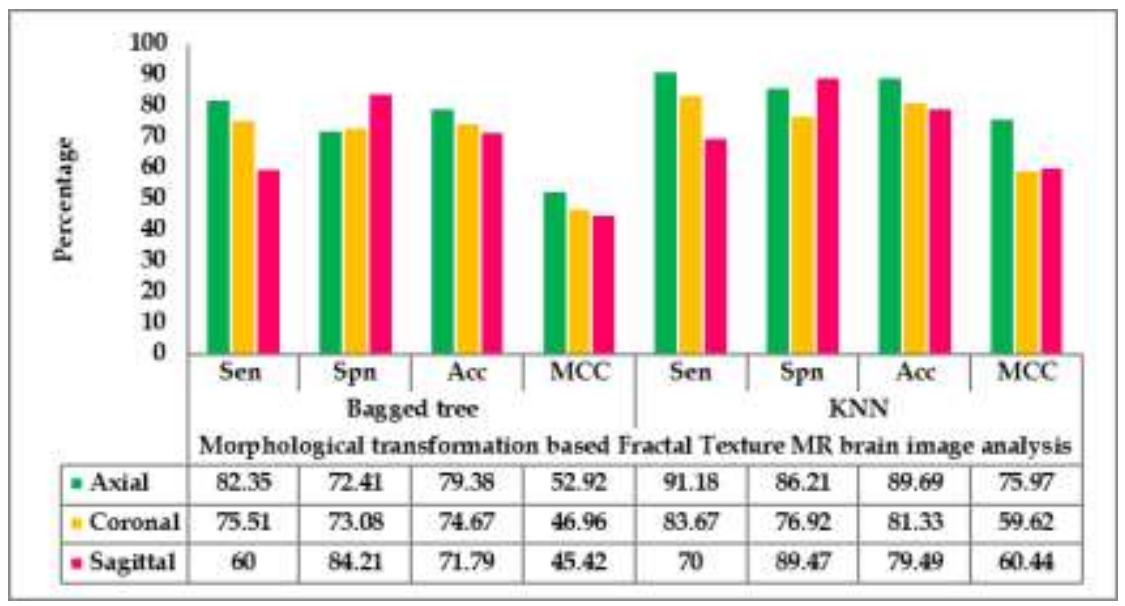

Figure 6. Morphological transformation based fractal texture analysis results
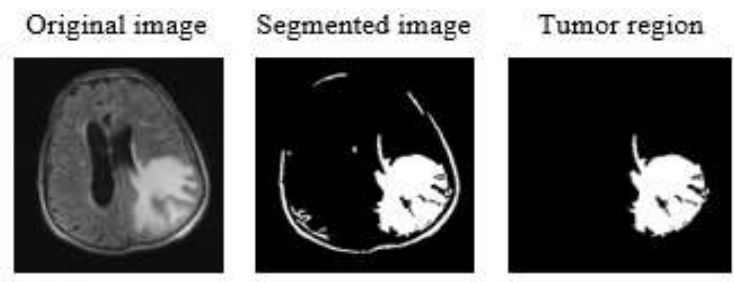

Tumor cell computation

Tumor Area $=6189$

Defected cells in a brain $=20.77 \mathrm{~mm}^{2}$

Figure 7. Tumor cells computation of sample axial view in MR brain image

\section{COMPARISON OF TRADITIONAL SFTA AND MODIFIED FRACTAL TEXTURE IMAGE ANALYSIS APPROACH}

In this section, the morphological transformation based fractal texture analysis approach has compared and discussed with the conventional SFTA technique. The comparison of these approaches are started with the extraction of threshold value, image decomposition and classification results. Initially, the extractions of threshold value of each approach are shown in Table 3 correspondingly.

Table 3. Sample extracted threshold value for different views in MR brain image decomposition in

\begin{tabular}{|c|c|c|c|c|c|}
\hline Image named & Image & Image View & Threshold position & SFTA & Proposed \\
\hline \multirow{2}{*}{ Sample1 } & & & $\mathrm{T}(1)$ & 0.1172 & 0.5352 \\
\hline & & & $\mathrm{T}(2)$ & 0.2949 & 0.7617 \\
\hline \multirow[t]{3}{*}{ Sample2 } & & Coronal & $\mathrm{T}(1)$ & 0.1133 & 0.2324 \\
\hline & & & $\mathrm{T}(2)$ & 0.2734 & 0.6133 \\
\hline & & & $\mathrm{T}(1)$ & 0.1074 & 0.2676 \\
\hline Sample3 & & & $\mathrm{T}(2)$ & 0.2676 & 0.6465 \\
\hline
\end{tabular}

As shown in above Figure 8, the conventional SFTA technique does not provide significant details more relevant to abnormal tissue of MR brain in three views of sample abnormal slices. But, the SFTA 
approach enables the minimum of abnormal tissue in sagittal view of abnormal slices at two threshold binary decomposition (TTBD). However, it needs the numerous number of image decomposition to extract the affected parts of the brain. Comparatively, proposed approach given the clear image details regarding to affected parts of brain tissues with limited number of image decomposition like STBD (single threshold binary decomposition). The SFTA approach takes maximum three or four level image decomposition to achieve the extraction of diseased tissues. But, this proposed approach provides better results minimum of single threshold and maximum of threshold value as 2. As mentioned in Figure 8, the proposed approach achieves better segmentation in minimum of single threshold position of $\mathrm{T}$ (1).

Subsequently, the extracted SFTA feature based classification for MR brain image analysis has illustrated in Figure 9. From the comparison of SFTA classification prediction results with the proposed approach of Morphological transformation based fractal texture image analysis as mentioned in Figure 6, the proposed approach also has superior results in the classification results in terms of accuracy, sensitivity, specificity, and MCC measures.

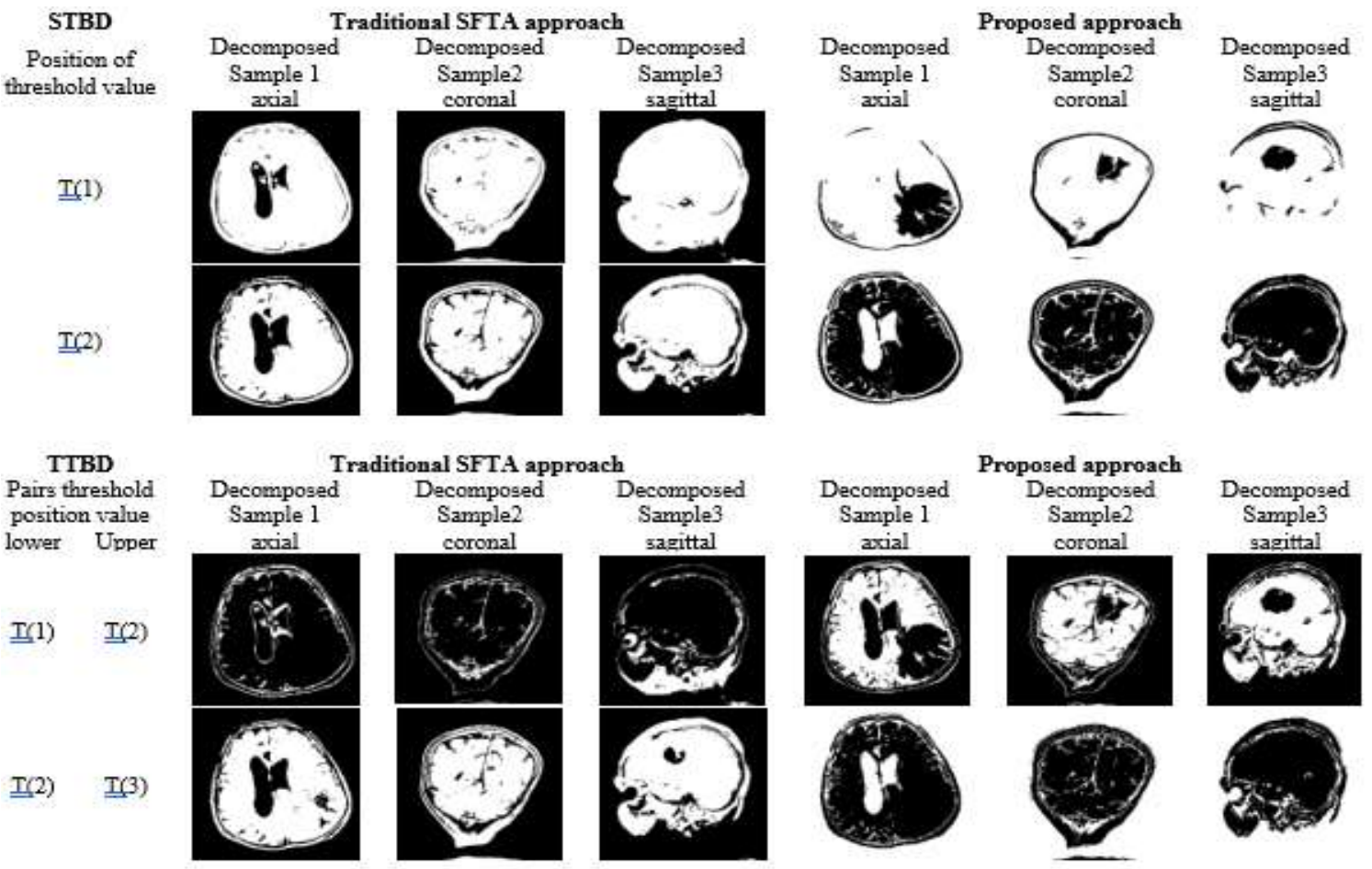

Figure 8. Sample decomposed different views of MR brain image outcomes in distinct approaches

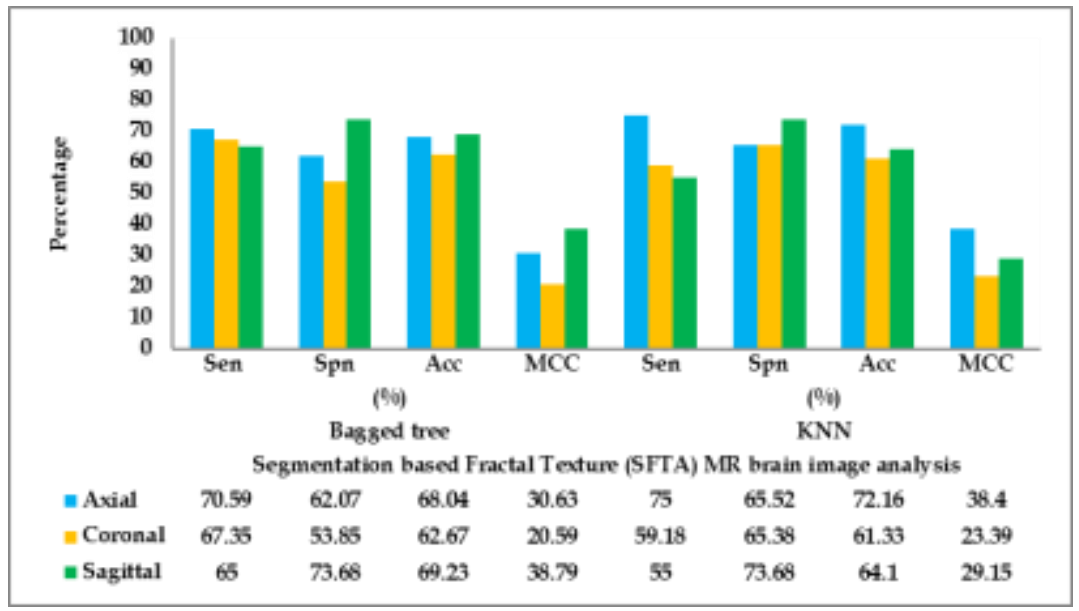

Figure 9. SFTA feature based classification of different views in MR brain image analysis 


\section{CONCLUSION}

This paper presents the morphological transformation based fractal texture image analysis of MR brain using KNN and ensemble bagged tree classifiers. This proposed technique provides the very promising outcomes to detect and classify the different views of normal and abnormal MR brain slices. The classification of bagged tree with use of extracted features accomplishes the accuracy of 79.38\%, 74.67\%, and $71.79 \%$ are axial, coronal, and sagittal view respectively. Similarly, the percentage of accuracy in the classification of KNN achieves the maximum of $89.69 \%$ for axial, $81.33 \%$ for coronal, and $79.49 \%$ for sagittal view rather than the ensemble bagged tree classifier. Finally, this proposed approach has been compared with conventional fractal texture analysis of SFTA in terms of size of threshold value, number of image decomposition, and the size of feature vector to analyse the MR brain images. From this comparison, the proposed approach achieves the highest percentage in accuracy, prediction of both positive and negative data with the minimum number of threshold value, less number of binary image decomposition.

\section{REFERENCES}

[1] Hayar Ayad, Mohammed Hasan Abdulameer, Loay E. George and Nidaa F. Hassan, "Descriptor Trends in Texture Classification for Material Recognition," Research Journal of Applied Science, Engineering and Technology, ISSN: 2040-7467, vol. 10, no.10, pp. 1089-1101, Aug 2015.

[2] Jong Kook Kim and Hyun Wook Park, "Statistical Textural Features for Detection of Microcalcifications in Digitized Mammograms," IEEE Transactions on Medical Imaging, vol. 18, no. 3, pp. 231-238, 1999.

[3] Amulya E R, Soumya Varma, and Vince Paul, "Classification of Brain MR Images using Texture Feature Extraction," International Journal of Innovative Research in Science Engineering and Technology, ISSN: 23198753, vol. 6, no. 4, pp. 7184-7190, 2017.

[4] Pratima Purushottam Gumaste, Vinayak K. Bairagi, "A Hybrid Method for Brain Tumor Detection using Advanced Textural Feature Extraction," Biomedical and Pharmacology Journal, vol. 13, no. 1, pp. 145-157, 2020.

[5] Marwan A. A. Hamid, and Najeed Ahmed Khan, "Investigation and Classification of MRI Brain Tumors Using Feature Extraction Technique," Springer Journal of Medical and Biological Engineering, vol. 40, pp. 307-317, Mar 2020.

[6] A. F. Costa, G. Humpire-Mamani and A. J. M. Traina, "An Efficient Algorithm for Fractal Analysis of Textures," 2012 25th SIBGRAPI Conference on Graphics, Patterns and Images, Ouro Preto, pp. 39-46, 2012 doi: 10.1109/SIBGRAPI.2012.15.

[7] Anju Krishna. M., Deepesh Edwin, and S. Hariharan, "Liver Tumor Segmentation and Feature Extraction using Segmentation based Fractal Textural Analysis Method," International Journal of Advanced Scientific Technologies, Engineering and Management Sciences, ISSN: 2454-356X, vol. 3, no. 1, pp. 169-172, Apr 2017.

[8] Spandana Paramkusham, Kunda M.M. Rao, B.V.V.S.N Prabhakar Rao, "Comparison of Raotation Invariant Local Frequency, LBP and SFTA methods for Breast Abnormality Classification," International Journal of Signal and Imaging Systems Engineering, vol. 11, no. 3, pp. 136-150, Jan 2018.

[9] Saban Ozturk, Bayram Akdemir, "Application of Feature and Classification Methods for Histopathological Image using GLCM, LBP, LBGLCM, GLRLM and SFTA,” Procedia Computer Science, ISSN: 1877-0509, vol. 132, pp. 40-46, June 2018.

[10] Jude Hemanth, D., and J. Anitha, "Image Pre-processing and Feature Extraction Techniques for Magnetic Resonance Brain Image Analysis," Springer, Berlin, Heidelberg, Computer Applications for Communication, Networking, and Digital Contents, vol. 350, pp. 349-356, Jan 2012.

[11] Marcin Iwanowski, Slawomir Skoneczny, Jaroslaw Szostakowski, "Image Features Extraction using Mathematical Morphology," Proceedings of SPIE-The International Society for Optical Engineering, pp. 01- 09, Oct 1997.

[12] Richard Alan Peters, "A New Algorithm for Image Noise Reduction using Mathematical Morphology," IEEE Transaction on Image Processing, vol. 4, no. 5, pp. 554-568, May 1995.

[13] R. Usha, and K. Perumal, "Morphological Transformation Based Fractal Texture Segmentation of MR Brain Tumor Slices," International Journal of Application or Innovation in Engineering and Management, ISSN: 23194847, vol. 8, no. 1, pp. 01- 07, Jan 2019.

[14] G. Bala Maalinii and Anand Jatti, "Brain Tumour Extraction using Morphological Reconstruction and Thresholding," https://doi.org/10.1016/j.matpr.2017.12.350 Materials Today: Proceedings, vol. 5, no. 4, pp. 10689-10696, 2018.

[15] S. Naganandhini, and P. Shanmugavadivu, "Threshold and Morphological Operations Based Brain Image Segmentation," International journal of Innovative Technology and Exploring Engineering (IJITEE), ISSN: 22783075, vol. 8, no. 6S3, pp. 96-100, April 2019.

[16] Andrei C. Jalba, Michael H.F., Wilkinson, Jos B.T.M. Roerdink, "Morphological Hat-transform Scale Spaces and their use in Pattern Classification," The Journal of Pattern Recognition, vol. 37, pp. 901-915, 2004.

[17] Suvarna D. Kamble, Pawar D.D., and Sharadkumar Jagtap, "Morphological Image Segmentation by Morphological Watersheds," International journal of Recent Scientific Research (IJRSR), ISSN: 0976-3031, vol. 10, no. 1, pp. 30370-30374, Jan 2019.

[18] Ankur Jyoti Das, Lipi B. Mahanta, and Vijay Prasad, "Automatic Detection of Brain Tumor from MR Images using Morphological Operations and K-means Based Segmentation," Proceedings of the Second International Conference on Emerging Research in Computing, Information, Communication and Applications (ERCICA) 2014, ISBN: 9789351072621 @ ELSEVIER Publication, vol. 2, pp. 845-850, 2014. 
[19] Radhakrishnan R, Teo Lay Lian, and B.S. Daya Sagar, "Estimation of fractal dimension through morphological decomposition," ELSEVIER, Chaos, Solitons and Fractals, vol. 21, pp. 563-572, July 2004.

[20] Yanpeng Wu, Xiaoqi Peng, Kai Ruan, Zhikun Hu, "Improved Image Segmentation Method based on Morphological Reconstruction," Springer (C) Multimedia Tools and Applications, vol. 76, pp. 19781-19793, Oct 2017.

[21] Yingbo Liang and Jian Fu, "Watershed Algorithm for Medical Image Segmentation Based on Morphology and Total Variation Model," International journal of Pattern Recognition and Artificial Intelligence, 1954019, vol. 33, no. 5, Jan 2019.

[22] Yu Wang, Fuqian Shi, Luying Cao, Nilanjan Dey, Qun Wu, Amira Salah Ashour, Robert Simon Sherratt, Venkatesan Rajinikanth, and Lijun Wu, "Morphological Segmentation Analysis and Texture-based Support Vector Machines Classification on Mice Liver Fibrosis Microscopic Images," Current Bioinformatics, ISSN: 2212-392X, vol. 14, no. 4, pp. 282-294, 2019.

[23] Dhananjay Bhaskar, DArrick Lee, Hildur Knutsdottir, Cindy Tan, Mohan Zhang, Pa,ela Dean, Calvin Roskelley, and Leah Edelstein-Keshet, "A Methodology for morphological Feature Extraction and Unsupervised Ccell Classification,” PLOS, 623793, pp. 01-30, April 2019.

[24] Nelson Luis Duranona Sosa, Jose Luis Vazquez Noguera, Juan Jose Caceres Silva, Miguel Garcia Torres, and Horacio Legal-Ayala, "RGB Inter-Channel Measures for Morphological Color Texture Characterization," Symmetry, vol. 11, pp. 01-16, Sep 2019.

[25] Usha R, Perumal K, "Image Enhancement of Medial Images Based on an Efficient Approach of Morphological and Arithmetic Operations," International Journal of Computer Trends and Technology (IJCTT), ISSN: 2231-2803, vol. 49, no. 5, pp. 253-258, July 2017.

[26] K. Ali, A. Jalil, M. U. Gull and M. Fiaz, "Medical Image Segmentation Using H-minima Transform and Region Merging Technique," 2011 Frontiers of Information Technology, Islamabad, pp. 127-132, 2011. doi: 10.1109/FIT.2011.31.

[27] Latourrette M, "Toward an Explanatory Similarity Measure for Nearest-Neighbor Classification," In: ECML '00: Proceedings of the 11th European Conference on Machine learning, London, UK, Springer-Verlag, Heidelberg, Lecture Notes in Computer Science @ Springer, vol. 1810, pp. 238-245, Jan 2003.

\section{BIOGRAPHIES OF AUTHORS}

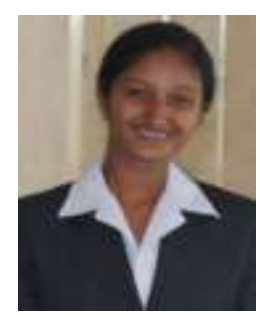

Mrs. R. Usha is pursuing Ph.D in the Department of Computer Applications, School of Information Technology, and Madurai Kamaraj University, India. She has completed M.Phil in Computer Applications at Madurai Kamaraj university, India in 2014. She has Contributed papers more than 8 in International Journals, National and International Conferences. Her Research work is focused on Medical Image Processing.

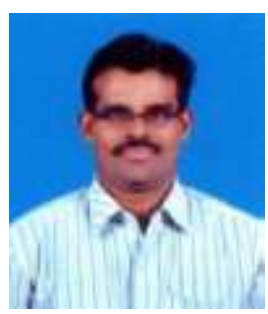

Mr. K. Perumal [2] is working as a Professor in the Department of Computer Applications, School of Information Technology, Madurai Kamaraj university, Madurai. He has contributed more than 35papers in International Journals and Conferences. He has Guiding 6 Scholars. His Interest includes Data mining, Image Processing, and Medical Imaging. 